\title{
Efficient high-energy photon production in the supercritical QED regime
}

\author{
Matteo Tamburini $\oplus^{1, *}$ and Sebastian Meuren $\circledast^{2,3, \uparrow}$ \\ ${ }^{1}$ Max-Planck-Institut für Kernphysik, Saupfercheckweg 1, D-69117 Heidelberg, Germany \\ ${ }^{2}$ Stanford PULSE Institute, SLAC National Accelerator Laboratory, Menlo Park, California 94025, USA \\ ${ }^{3}$ Department of Astrophysical Sciences, Princeton University, Princeton, New Jersey 08544, USA
}

(Received 18 December 2019; revised 24 August 2020; accepted 1 October 2021; published 5 November 2021)

\begin{abstract}
When dense high-energy lepton bunches collide, the beam particles can experience rest-frame electromagnetic fields which greatly exceed the QED critical one. Here it is demonstrated that beamstrahlung efficiently converts lepton energy to high-energy photons in this so-called supercritical QED regime, as the single-photon emission spectrum exhibits a pronounced peak close to the initial lepton energy. It is also shown that the observation of this high-energy peak in the photon spectrum requires one to mitigate multiple photon emissions during the interaction. Otherwise, the photon recoil induces strong correlations between subsequent emissions which soften the photon spectrum and suppress the peak. The high-energy peak in the photon spectrum constitutes a unique observable of photon emission in the supercritical QED regime and provides decisive advantages for the realization of an efficient multi-TeV laserless gamma-gamma collider based on electron-electron collisions.
\end{abstract}

DOI: 10.1103/PhysRevD.104.L091903

A future multi-TeV lepton collider has to be linear in order to mitigate energy losses via synchrotron radiation [1]. As two colliding bunches cross only once in a linear collider, extremely high particle densities are necessary at the interaction point in order to achieve the luminosities required to search for physics beyond the standard model [2-4]. As a result, beamstrahlung energy losses become a decisive limiting factor, especially in the multi-10-TeV regime [5-9].

Beamstrahlung is primarily characterized by the quantum parameter $\chi=\Upsilon=F^{*} / F_{\text {cr }}$ [8], where $F^{*}$ denotes the electric field in the rest frame of a beam particle and $F_{\text {cr }}=m^{2} c^{3} /|e| \hbar \approx 1.3 \times 10^{18} \mathrm{~V} / \mathrm{m}$ is the QED critical (Schwinger) field $\left(F_{\mathrm{cr}} / c \approx 4.4 \times 10^{9} \mathrm{~T}\right)$. For $\chi \ll 1$ the radiative energy loss of an unbound ultrarelativistic charge is well approximated by the prediction of classical electrodynamics, whereas for $\chi \gtrsim 1$ quantization effects in the radiation field become essential [10-12]. While the regime $\chi \lesssim 1$ is relatively well explored theoretically $[10,12]$ (see [13-16] for recent experiments), the supercritical regime $\chi \gg 1$ is still poorly understood. When $\alpha \chi^{2 / 3} \gtrsim 1\left(\chi \gtrsim 10^{3}\right)$, where $\alpha=e^{2} /\left(4 \pi \epsilon_{0} \hbar c\right) \approx 1 / 137$ is the fine-structure constant, radiative corrections become significant [10], and even

\footnotetext{
*matteo.tamburini@mpi-hd.mpg.de

smeuren@stanford.edu
}

Published by the American Physical Society under the terms of the Creative Commons Attribution 4.0 International license. Further distribution of this work must maintain attribution to the author(s) and the published article's title, journal citation, and DOI. Funded by SCOAP ${ }^{3}$. a complete breakdown of perturbation theory has been conjectured [17,18] (see also [19-23] for recent theoretical studies and [24-28] for proposals to probe this regime experimentally).

State-of-the-art linear lepton collider designs such as CLIC and ILC [29,30] employ long and flat bunches in order to minimize beamstrahlung. Recently, it was suggested in Ref. [24] that beamstrahlung could also be mitigated by colliding short and round bunches and operating in the supercritical QED regime $(\chi \gg 1)$. A different approach, which completely circumvents the beamstrahlung problem, are gamma-gamma colliders [6,31-33].

The state-of-the-art concept to generate high-energy photons for a gamma-gamma collider is based on Compton backscattering of two intense laser pulses with two counterpropagating lepton bunches, properly coordinated in space and time [6,32-34]. However, Compton backscattering becomes increasingly more challenging to realize with increasing center-of-mass energy [6,33]. Remarkably, beamstrahlung itself could be used to produce high-energy gamma photons $[35,36]$.

Here we point out that qualitatively new features appear in the photon emission spectrum in the supercritical QED regime. In fact, we demonstrate that (i) for $\chi \gtrsim 16$ the probability for an electron to emit a single photon carrying almost all its initial energy strongly increases; (ii) the single-emission photon spectrum can be observed in asymmetric electron-electron beam collisions and provides direct quantitative in situ information on the average $\chi$ of the beam achieved during the collision; (iii) when multiple photon emissions become dominant, the high-energy peak 


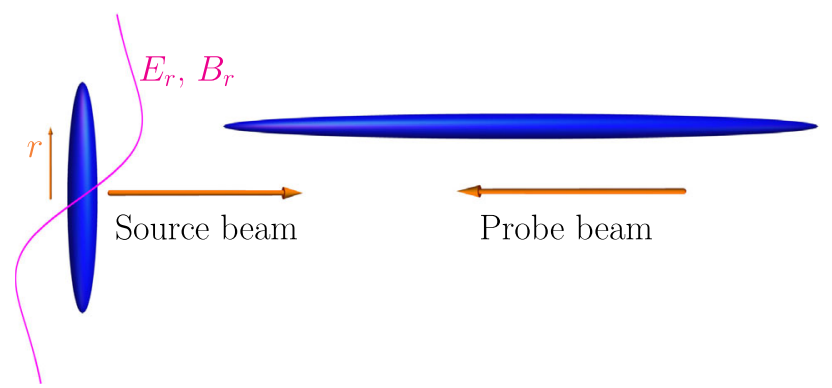

FIG. 1. Schematic setup. A pancake-shape dense source beam collides with an elongated cigar-shape low-density probe beam. The probe beam collides with a transverse impact parameter $r$, such that the source beam electric $E_{r}$ and magnetic $B_{r}$ fields approach their maximum.

in the total and, remarkably, even in the single-photon emission spectrum vanishes.

On one hand, these findings are of intrinsic interest for both fundamental science and practical applications. In fact, they provide a unique observable which, on a shot-toshot basis, directly informs on the actually achieved average $\chi$ of the beam, therefore overcoming experimental uncertainties such as jitter in the collision parameters. On the other hand, they reveal an optimal regime for efficient high-energy photon production. This optimal regime is attained by properly shaping the colliding bunches such that most of the electrons of the beam reach $\chi \gg 1$ and emit once, while further emissions by the same beam electrons remain negligible. This allows one to greatly increase the yield of photons that carry nearly all the energy of the emitting electron while simultaneously suppressing the lowenergy photon background. This is a decisive advantage for a gamma-gamma collider as, during gamma-gamma collisions, low-energy photons can convert high-energy photons into $e^{-} e^{+}$pairs via the linear Breit-Wheeler process, therefore substantially reducing the luminosity of high-energy photons.

In the following we consider an asymmetric electronelectron collider setup (see Fig. 1). A short dense pancakeshape electron "source" beam collides head on with an elongated cigar-shape high-energy "probe" beam. For clarity, source and probe-beam parameters are denoted with the subscript $s$ and $p$, respectively. As the electromagnetic field experienced by the probe beam particles changes significantly as a function of the impact parameter, the considered asymmetric setup avoids a trivial average over different values of $\chi_{p}$, which is always present in symmetric collisions. In comparison with symmetric collisions the "source" beam provides the strong field; i.e., its longitudinal bunch length $\left(\ell_{s}\right)$, transverse rms size $\left(\sigma_{s}\right)$, and number of electrons $\left(N_{s}\right)$ are relevant for calculating the field. On the other hand, the high-energy "probe" beam provides the large gamma factor $\gamma_{p}$ to boost the experienced rest-frame field. Note that the energy of the source beam is not relevant for attaining large $\chi_{p}=\gamma_{p} \sqrt{\left(\boldsymbol{E}_{s}+\boldsymbol{v}_{p} \times \boldsymbol{B}_{s}\right)^{2}-\left(\boldsymbol{E}_{s} \cdot \boldsymbol{v}_{p} / c\right)^{2}} / F_{\mathrm{cr}}$, where $\boldsymbol{E}_{s}$ $\left(\boldsymbol{B}_{s}\right)$ is the electric (magnetic) field of the source beam and $v_{p}\left(\gamma_{p}\right)$ is the velocity (relativistic factor) of the probe beam in the laboratory frame. In fact, the quantum parameter $\chi_{p}$ can also be expressed directly in terms of the beam parameters [8]:

$$
\chi_{p} \sim \gamma_{p} \alpha N_{s} \frac{\chi_{c}^{2}}{\sigma_{s} \ell_{s}} \text {, with } \chi_{c}=\frac{\hbar}{m c} \approx 3.9 \times 10^{-13} \mathrm{~m} .
$$

For the beam parameters considered here, the source beam remains almost unaffected by the interaction with the probe beam (see below and Ref. [8]). Furthermore, the interaction is collisionless; i.e., the probe beam interacts only with the collective electromagnetic field of the source beam. As the probe beam is ultrarelativistic, the quasiclassical approximation holds; i.e., particle trajectories are calculated using classical electrodynamics, whereas the emission itself is calculated quantum mechanically [10-12]. The formation length for photon emission scales as (assuming $\chi \gg 1$ ) [37]

$$
l_{f} \sim \frac{\gamma \chi_{c}}{u^{1 / 3} \chi^{2 / 3}}, \quad u=\frac{\omega}{1-\omega}, \quad \omega=\frac{\varepsilon_{\gamma}}{\varepsilon} .
$$

For photon energies $\varepsilon_{\gamma}$ comparable to the initial energy $\varepsilon=\gamma m c^{2}$ of the emitting electron $(u \gtrsim 0.1)$ and for the beam parameters considered here $\left(\gamma \sim 10^{5}, \chi \sim 100\right.$, $\ell_{s} \sim 100 \mathrm{~nm}$ ), the formation length is much shorter than the scale on which the electromagnetic field of the source beam changes $\left(l_{f} \ll \ell_{s}\right)$. Furthermore, the electron energy is negligibly altered during the emission process as $\left|e \boldsymbol{E}_{s}\right| l_{f} \sim m c^{2}(\chi / u)^{1 / 3} \ll \gamma m c^{2}$. Thus, the source-beam field is locally constant during the photon emission process [38-40], which allows one to employ the differential radiation probability in a constant homogeneous field, where the local value of $\chi$ and $\gamma$ is used $[8,10,11]$ :

$$
\begin{aligned}
\frac{d^{2} W}{d t d \omega}= & \frac{\alpha}{\sqrt{3} \pi \tau_{c} \gamma}\left\{\left[2+\frac{\omega^{2}}{(1-\omega)}\right] \mathrm{K}_{2 / 3}\left[\frac{2 \omega}{3 \chi(1-\omega)}\right]\right. \\
& \left.-\int_{2 \omega /[3 \chi(1-\omega)]}^{\infty} d y \mathrm{~K}_{1 / 3}(y)\right\} .
\end{aligned}
$$

Here $\tau_{c}=\hbar /\left(m c^{2}\right)$ is the Compton time, and $\mathrm{K}_{\nu}(x)$ is the modified Bessel function of the second kind [41]. Correspondingly, the local emission rate is $d W / d t=$ $\int_{0}^{1} d \omega d^{2} W / d t d \omega$, while the normalized emitted power is $d I / d t=\int_{0}^{1} d \omega d^{2} I / d t d \omega$, where $d^{2} I / d t d \omega=\omega d^{2} W / d t d \omega$.

A detailed analysis of Eq. (3) reveals a distinctive feature of the photon emission spectrum which occurs exclusively in the supercritical regime. Whereas $d^{2} W / d t d \omega$ is a monotonically decreasing function of $\omega$ for $\chi \lesssim 16$, it develops a local minimum and maximum for $\chi \gtrsim 16$, which 


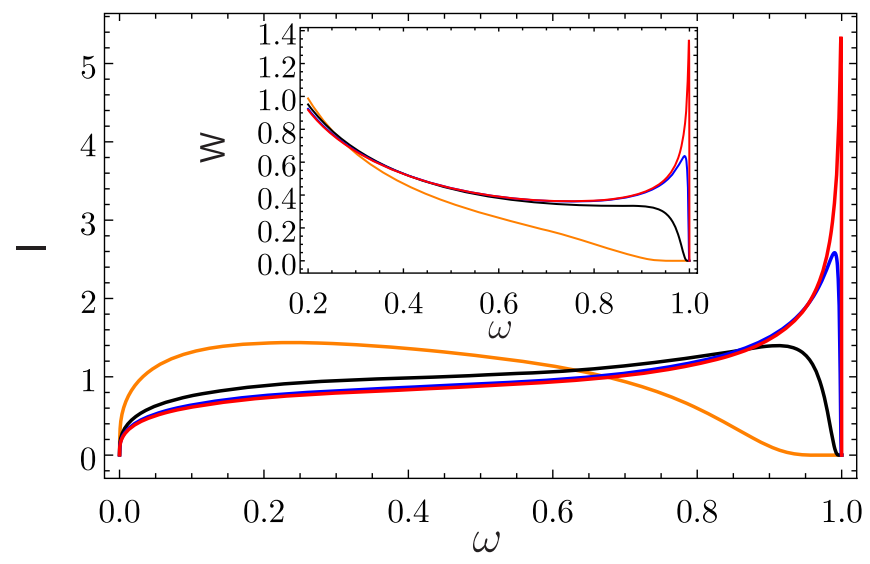

FIG. 2. Normalized photon emission spectrum $\mathrm{I}=$ $d^{2} I / d t d \omega(d I / d t)^{-1}$ and normalized probability $\mathrm{W}=$ $d^{2} W / d t d \omega(d W / d t)^{-1}$ (inset) for $\chi=1.6$ (orange lines), $\chi=$ 16 (black lines), $\chi=160$ (blue lines), and $\chi=1600$ (red lines).

results in a peak close to $\omega=1$ (see inset of Fig. 2 and Refs. $[42,43]$ ). For $\chi>16$ the minimum and maximum are approximately located at, respectively,

$$
\begin{aligned}
& \omega_{\min } \approx 0.754+\frac{(15.7+0.146 \chi)}{\chi^{2}}, \\
& \omega_{\max } \approx 1-\frac{(174+20 \chi)}{15 \chi^{2}} .
\end{aligned}
$$

The height of the peak $H$ is given by

$$
H=\frac{\left(d^{2} W / d t d \omega\right)\left(\omega_{\max }\right)}{\left(d^{2} W / d t d \omega\right)\left(\omega_{\min }\right)} \approx \frac{1.315+0.315 \chi}{\chi^{2 / 3}}
$$

and provides a unique observable of the average $\chi$ that is actually achieved during the interaction. Note that the peak at $\omega \approx 1$ originates from the factor $(1-\omega)^{-1}$ and guarantees that Eq. (3) conserves energy even in the deep quantum regime $\chi \gg 1$. In fact, photon emission with $\varepsilon_{\gamma}>\varepsilon$ is impossible within the approximation that the energy transferred by the field during the emission itself is negligible.

Figure 2 displays the normalized emission spectrum $\mathrm{I}=$ $d^{2} I / d t d \omega(d I / d t)^{-1}$ and the normalized photon emission probability $\mathrm{W}=d^{2} W / d t d \omega(d W / d t)^{-1}$ in four different regimes: (i) the critical regime ( $\chi \sim 1$, orange line), (ii) transition between the critical and the supercritical regime $(\chi \sim 10$, black line), (iii) the supercritical regime $(\chi \sim 100$, blue line), and (iv) the fully nonperturbative regime $\left(\alpha \chi^{2 / 3} \sim 1\right.$, red line). Whereas electrons still emit in a broad energy range for $\chi \sim 1$, a sharp peak close to the initial electron energy $(\omega \approx 1)$ appears in the supercritical regime $(\chi>16)$. The probability of producing a photon with energy beyond $\omega_{\min }$ already exceeds $9 \%$ for $\chi>60$ and basically saturates to approximately $11 \%$ for $\chi \gtrsim 800$. However, the height of the peak monotonically increases with increasing $\chi$; i.e., the photon spectrum at $\omega \approx 1$ becomes more and more monochromatic [see Eq. (5) and Fig. 2].

In order to quantitatively investigate the photon emission spectrum, 3D Monte Carlo simulations of beam-beam collision were performed using the state-of-the-art methodology [44-46]. In the simulations an exact 3D analytical solution of Maxwell's equations was used for the electromagnetic fields of the source beam (see Supplemental Material [47]). The source beam has $10 \mathrm{GeV}$ energy, $0.96 \mathrm{nC}$ charge, $\ell_{s}=100 \mathrm{~nm}$ bunch length (in the laboratory frame), and $\sigma_{s}=300 \mathrm{~nm}$ transverse size. In the laboratory, a maximum electric (magnetic) field of $E_{\max } \approx$ $2.6 \times 10^{14} \mathrm{~V} / \mathrm{m}\left(B_{\max } \approx 8.6 \times 10^{5} \mathrm{~T}\right)$ is achieved at an impact parameter of $r \approx 500 \mathrm{~nm}$. The source beam parameters considered here are comparable to those achievable at the FACET-II facility at SLAC [48].

The probe beam has $100 \mathrm{GeV}$ energy with $100 \mathrm{MeV}$ energy spread, $16 \mathrm{pC}$ charge, $\ell_{p}=300 \mu \mathrm{m}$ bunch length, and $\sigma_{p}=50 \mathrm{~nm}$ transverse size. As a result, the maximum electric (magnetic) field of the probe beam $E_{\max } \approx 8.7 \times$ $10^{9} \mathrm{~V} / \mathrm{m}\left(B_{\max } \approx 29 \mathrm{~T}\right)$ at $r \approx 80 \mathrm{~nm}$ is much weaker and its density is much lower than the corresponding values of the source beam. Thus, the source beam is basically unaffected by the interaction as both collisions and energy losses associated with the probe beam fields are negligible. Due to the finite transverse size of the probe beam $\chi_{p}$ ranges approximately from 75 to 77 . For the above parameters the average emission probability per electron is approximately 0.12 . Note that nanometer-scale beam stabilization and thus smaller than $1 \%$-level fluctuations in $\chi$ are anticipated for state-of-the-art final focusing systems [49-51]. In addition, each electron emits on average less than once and photon emission occurs in a cone with $1 / \gamma_{p} \approx 5 \mu \mathrm{rad}$ opening angle around the propagation direction of the emitting electron [11,52]. Hence, the angular distribution of the emitted photons is to excellent accuracy the same as the electron beam angular distribution.

Figure 3(a) reports the photon energy distribution for electrons which emitted only one (two) photon(s) during the interaction [orange (black) line] and the total photon distribution (blue line). Accordingly, the photon spectrum is dominated by single emissions while secondary and higher-order processes are suppressed (see also the inset of Fig. 4). The peak height $H$ obtained from simulations (total spectrum, blue line) corresponds to $\chi_{p} \approx 69$ if Eq. (5) is employed. This value is within the $10 \%$ error margin which we expect due to the presence of multiple emissions. In fact, $H$ provides a lower bound to $\chi_{p}$, which converges to the actual value in the single-emission limit.

Next, we consider the same parameters as above but increase (decrease) the source beam length (transverse size) by a factor of 25 , i.e., employ $\ell_{s}=2500 \mathrm{~nm}$ and $\sigma_{s}=12 \mathrm{~nm}$. This scaling leaves $\chi_{p}$ invariant [see Eq. (1)], which is now reached at an impact parameter $r \approx 20 \mathrm{~nm}$. 


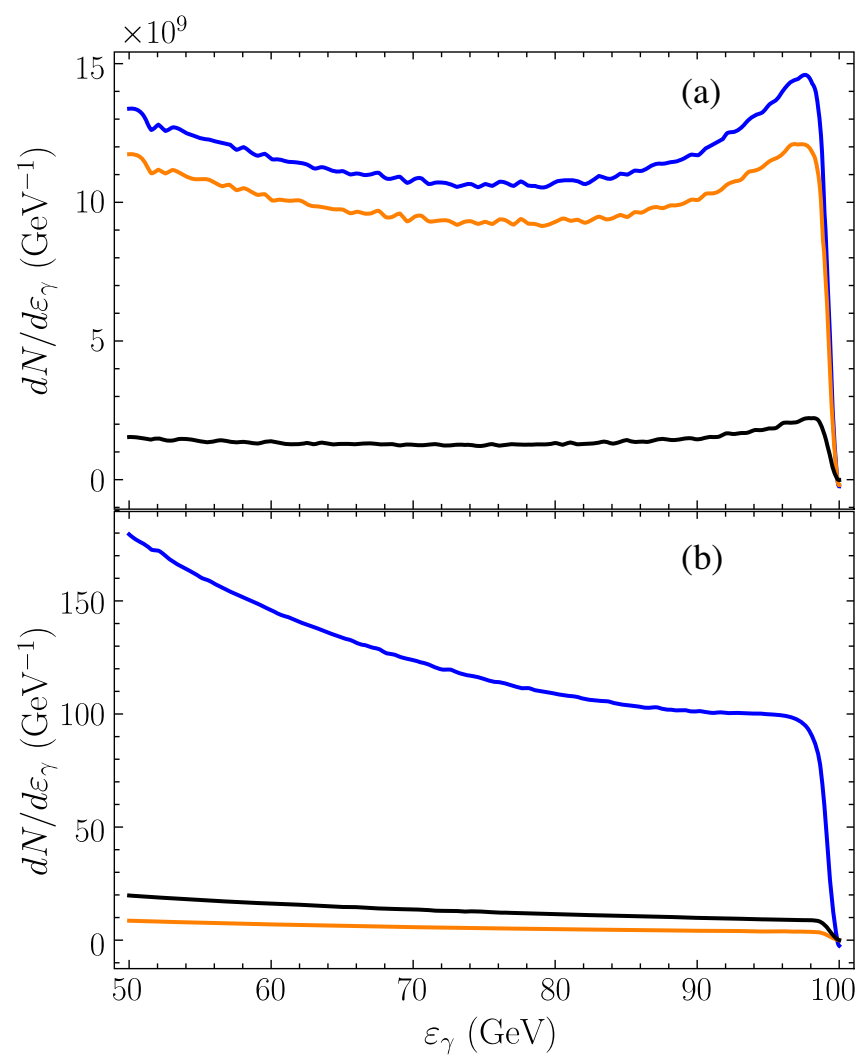

FIG. 3. Photon emission probability. The solid orange, black and blue lines report photons that originate from electrons that emitted only once, only twice, and all final photons, respectively. (a) Short interaction time $\left(\ell_{s}=100 \mathrm{~nm}\right)$, single emissions dominant; (b) long interaction time $\left(\ell_{s}=2500 \mathrm{~nm}\right)$, multiple emissions dominant. See the main text for further details.

To keep the variation of $\chi_{p}$ comparable to the first simulation, we also reduce the transverse size of the probe beam to $\sigma_{p}=2 \mathrm{~nm}$. As a result, the average number of photon emissions per electron increases to approximately 3.0

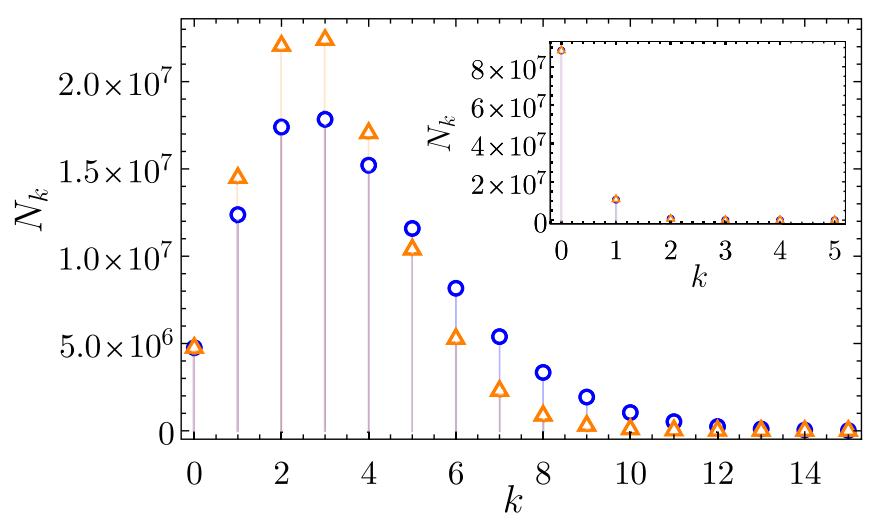

FIG. 4. Number of probe beam electrons $N_{k}$ that emitted $k$ photons in the interaction with the source beam. Blue circles report the simulation results, orange triangles the Poisson prediction. Main plot: $\ell_{s}=2500 \mathrm{~nm}$. Inset: $\ell_{s}=100 \mathrm{~nm}$. [see Fig. 3(b) and Fig. 4]. In contrast to the previous simulation, the spectrum no longer exhibits a peak.

In order to explain this transition, we assume that a probe particle experiences the supercritical quantum regime $\left(\chi_{p} \gg 1\right)$. As shown in Fig. 2, it is likely that this particle emits a hard photon with $\omega \approx 1$. Due to the large recoil, the probe particle has a much lower energy $\varepsilon^{\prime}=\varepsilon(1-\omega)$ after the emission. In the regime $\chi_{p} \gg 1$, the scaling of the radiation probability $W_{p} \sim\left[\ell_{s} /\left(\chi_{c} \gamma_{p}\right)\right]^{1 / 3}$ (see, e.g., Refs. $[8,10,24])$ implies that a particle with lower energy has an increased radiation probability. Therefore, the emission of a hard photon $(\omega \approx 1)$ increases the probability to emit a second photon, which is on average much softer. On the contrary, the emission of a soft photon $(\omega \ll 1)$ is less likely followed by a second emission. As a consequence, we expect the peak to vanish in the total photon spectrum when multiphoton emissions are dominant. Remarkably, the peak disappears also in the one-photon emission spectrum [see the orange line in Fig. 3(b)], which is naively not expected based on perturbation theory (see below).

In the following the photon emission distribution is calculated analytically. Assuming that the locally-constantfield approximation holds, the single-photon emission probability given in Eq. (3) is always applicable for sufficiently small time intervals $d t$. Thus, the probability $S\left(t, t^{\prime} ; \varepsilon\right)$ that an electron with energy $\varepsilon$ does not emit a photon during the time interval $\left[t, t^{\prime}\right]$ is given by (see Supplemental Material [47])

$$
S\left(t, t^{\prime} ; \varepsilon\right)=\exp \left[-\int_{t^{\prime}}^{t} d \tau \frac{d W}{d \tau}(\varepsilon, \tau)\right] .
$$

Correspondingly, the electron "decays" exponentially, with a radiative lifetime given by the total emission probability per unit time $d W(\varepsilon, t) / d t=\int_{0}^{\varepsilon} d \varepsilon^{\prime} d^{2} W\left(\varepsilon^{\prime}, \varepsilon, t\right) / d t d \varepsilon^{\prime}$. As $\varepsilon^{\prime}=\varepsilon(1-\omega)$, the quantities $d^{2} W / d t d \varepsilon^{\prime}$ and $d^{2} W / d t d \omega$ are trivially related [see Eq. (3)]. Note that $S\left(t, t^{\prime} ; \varepsilon\right)$ can also be derived from the radiatively corrected wave function of the electron (see [53-56] for further details).

The probability $\left(d P_{1} / d \varepsilon^{\prime}\right)\left(\varepsilon^{\prime}, t\right)$ that an electron has an energy within $\left[\varepsilon^{\prime}, \varepsilon^{\prime}+d \varepsilon^{\prime}\right]$ at time $t$ after radiating exactly one photon is

$$
\begin{aligned}
\frac{d P_{1}}{d \varepsilon^{\prime}}\left(\varepsilon^{\prime}, t\right)= & \int_{-\infty}^{t} d \tau S\left(t, \tau ; \varepsilon^{\prime}\right) \\
& \times \frac{d^{2} W}{d \tau d \varepsilon^{\prime}}\left(\varepsilon^{\prime}, \varepsilon_{i}, \tau\right) S\left(\tau,-\infty ; \varepsilon_{i}\right) .
\end{aligned}
$$

Here and in the following, $\varepsilon_{i}$ denotes the initial electron energy, we implicitly assume that the work performed by the external field is negligible compared to the electron energy, and $\chi(t)$ is obtained from the electron trajectory. Equation (7) explains the suppression of the peak even in the one-photon emission spectrum when multiple emissions become dominant (see Fig. 3). In fact, in the regime 
$\chi \gg 1$ substantial recoil is likely, which implies $\varepsilon^{\prime} \ll \varepsilon_{i}$. Correspondingly, the decay exponent after the emission $S\left(t, \tau ; \varepsilon^{\prime}\right)$ is substantially smaller than it would be with negligible recoil $S\left(t, \tau ; \varepsilon^{\prime} \approx \varepsilon_{i}\right)$; i.e., the electron "radiative lifetime" substantially decreases after the emission. Therefore, the high-energy part of the spectrum $\omega=1-$ $\varepsilon^{\prime} / \varepsilon_{i} \approx 1$ is suppressed for sufficiently long interaction time. Consequently, even the one-photon emission spectrum differs qualitatively from Eq. (3) [compare Figs. 3(a) and 3(b)]. Note that for short interaction times $S\left(t, \tau ; \varepsilon^{\prime}\right) \approx 1$, independently of the magnitude of the recoil [see Eq. (6)], and the spectrum coincides with Eq. (3).

Equation (7) can be easily generalized to $n$ photon emissions (see Supplemental Material [47] for further details)

$$
\begin{aligned}
\frac{d P_{n}}{d \varepsilon^{\prime}}\left(\varepsilon^{\prime}, t\right)= & \int_{-\infty}^{t} d \tau S\left(t, \tau ; \varepsilon^{\prime}\right) \\
& \times \int_{\varepsilon^{\prime}}^{\varepsilon_{i}} d \varepsilon \frac{d^{2} W}{d \tau d \varepsilon^{\prime}}\left(\varepsilon^{\prime}, \varepsilon, \tau\right) \frac{d P_{n-1}}{d \varepsilon}(\varepsilon, \tau) .
\end{aligned}
$$

In Eqs. (7) and (8) we have implicitly assumed that the local radiation probability $\left(d^{2} W / d \tau d \varepsilon^{\prime}\right)\left(\varepsilon^{\prime}, \varepsilon, \tau\right)$ depends only on the time $\tau$ at which the photon is emitted and on the electron instantaneous energy $\varepsilon$. However, the position of the electron and thus the instantaneous field strength depends, in general, on the full history of previous emissions and not just on $\tau$ and $\varepsilon$. This is a reasonable approximation when the particle is ultrarelativistic and the background field is transversely sufficiently homogeneous.

Finally, we are interested in the asymptotic probabilities $P_{n}$ that an electron has emitted exactly $n$ photons during the interaction:

$$
P_{n}=P_{n}(\infty), \quad P_{n}(t)=\int_{0}^{\varepsilon_{i}} d \varepsilon^{\prime} \frac{d P_{n}}{d \varepsilon^{\prime}}\left(\varepsilon^{\prime}, t\right)
$$

with $P_{0}(t)=S\left(t,-\infty ; \varepsilon_{i}\right)$. In the classical limit $(\chi \ll 1)$ the recoil is negligible; thus, $S\left(t, \tau ; \varepsilon^{\prime} \approx \varepsilon\right) S\left(\tau, t^{\prime} ; \varepsilon\right) \approx$ $S\left(t, t^{\prime} ; \varepsilon\right)$, and one finds that the number of emitted photons $P_{n}$ follows a Poissonian distribution (see Supplemental Material [47] and Refs. [57-59] for further details)

$$
P_{n}=\frac{\mathcal{W}^{n}}{n !} \exp (-\mathcal{W}), \quad\langle n\rangle=\sum_{n=0}^{\infty} n P_{n}=\mathcal{W} .
$$

Here, the decay exponent $\mathcal{W}=\int_{-\infty}^{+\infty} d \tau(d W / d \tau)\left(\varepsilon_{i}, \tau\right)$ factorizes, is independent of the number of emitted photons, and is constant across the spectrum. This is in sharp contrast to the $\chi \gg 1$ regime, where it is highly probable that $\varepsilon^{\prime} \ll \varepsilon$ such that $S\left(t, \tau ; \varepsilon^{\prime}\right) S\left(\tau, t^{\prime} ; \varepsilon\right) \neq S\left(t, t^{\prime} ; \varepsilon\right)$ and the decay exponent changes substantially when multiple photon emissions become probable, which results in a qualitative change of the energy distribution even for photons originating from electrons that emitted only once [see the orange line in Fig. 3(b)].

In Fig. 4 the simulated distribution (blue circles) is compared to the Poissonian prediction (orange triangles). For short interaction times (inset of Fig. 4) $P_{0}$ is dominant, and the Poissonian approximation is valid. However, when $\chi \gg 1$ and $P_{n>0}$ is dominant, substantial deviations are found (see the main plot of Fig. 4).

Finally, we consider the attainable luminosity of a gammagamma collider based either on beamstrahlung in the $\chi \gg 1$ regime or on Compton backscattering. The luminosity of two identical Gaussian photon bunches colliding head on is $\mathcal{L}=f_{c} N_{\gamma}^{2} / 4 \pi \sigma_{x} \sigma_{y}$, where $f_{c}$ is the bunch collision frequency, $N_{\gamma}$ the number of photons per bunch, and $\sigma_{x}$ and $\sigma_{y}$ the rms transverse bunch sizes. Both for beamstrahlung and for Compton backscattering, high-energy photon emission occurs in a cone with $1 / \gamma_{p}$ opening angle around the propagation direction of the emitting lepton. Hence, for the same probe electron beam and after ballistic propagation, the photon bunches generated with the two methods have similar $\sigma_{x}$ and $\sigma_{y}$ at collision. Also, $f_{c}$ is determined by the electron beam (and laser for Compton backscattering) repetition rate, which is assumed to be similar for both beamstrahlung and Compton backscattering. In this case, the decisive factor for achieving high luminosity is $N_{\gamma}$.

In a multi-TeV gamma-gamma collider higher-energy photons are of interest. For beamstrahlung in the $\chi \gg 1$ regime reached with a $1 \mathrm{TeV}$ probe beam and the same source beam as considered above, $\omega_{\min } \approx 0.75$ with the quasimonochromatic peak at $\omega_{\max } \approx 0.998$ and more than $11 \%$ photons with $\omega>\omega_{\min }$. For Compton backscattering the highest attainable photon energy is $\omega_{C, \max }=x / 1+x$, where $x=4 \varepsilon \varepsilon_{L} / m^{2} c^{4}$ and $\varepsilon_{L}$ is the incident photon energy [34]. In order to reach $\omega_{C, \max } \approx \omega_{\max } \approx 0.998, x \approx 500$ is required, which can be obtained by colliding $\varepsilon_{L} \approx 33 \mathrm{eV}$ photons generated by a free-electron laser with the $1 \mathrm{TeV}$ probe beam. However, for $x \gtrsim 4.8(x \gtrsim 8)$ the competing process $\gamma \gamma_{0} \rightarrow e^{-} e^{+}\left(e^{-} \gamma_{0} \rightarrow e^{-} e^{-} e^{+}\right)$, where $\gamma$ and $\gamma_{0}$ refer to the Compton-backscattered and incident photon, respectively, is not kinematically forbidden and has, in general, a larger cross section than the Compton one [6,34]. Therefore, traditional gamma-gamma collider designs focus on the regime $x \lesssim 4.8[6,34]$. Recently, however, research on high-energy photon production via Compton backscattering in the $x \gg 1$ regime has been pursued $[60,61]$. These proposals suggest to employ polarized laser pulses colliding with polarized electron beams to suppress backgrounds. Remarkably, because of the polarization dependence of the photon emission spectrum, strongly peaked emission with photon energy around $\omega_{\max }$ and drastic suppression of lower-energy photons is possible also for beamstrahlung in the $\chi \gg 1$ regime by employing a polarized probe electron beam (see Fig. 4 in Ref. [62]).

In conclusion, we have shown that in the supercritical QED regime the beamstrahlung spectrum exhibits a 
quasimonochromatic peak close to the energy of the emitting lepton and that the height of this peak provides direct quantitative in situ information on the quantum parameter $\chi$ achieved during the interaction. Moreover, we have shown that, due to the presence of strong correlations, this peak vanishes in a regime where multiphoton emissions become dominant. The recoil-induced correlations between different photon emissions manifest themselves in a photon statistic which significantly deviates from a Poissonian distribution. These results pave the way to a photon source that is capable of efficiently delivering above TeV-energy photons, a critical step toward realizing a multi-TeV photon-photon collider.

The authors would like to thank Fabrizio Del Gaudio, Antonino Di Piazza, Gerald Dunne, Nathaniel Fisch, Thomas Grismayer, Christoph Keitel, Michael Peskin, David Reis, Luís Silva, Glen White, Vitaly Yakimenko, and all participants of the 2019 SLAC workshop "Physics
Opportunities at a Lepton Collider in the Fully Nonperturbative QED Regime" for stimulating discussions. At SLAC, S. M. was supported by the U.S. Department of Energy under Contract No. DE-AC02-76SF00515. At Princeton, S.M. received funding from the Deutsche Forschungsgemeinschaft (DFG, German Research Foundation) under Grant No. 361969338. M. T. discovered that a peak appears in the photon emission probability in the supercritical QED regime for $\chi \gtrsim 16$ and studied its properties in 2014 and, in particular, obtained Eqs. (4) and (5) of the main text and Eqs. (S1)-(S2c) of Supplemental Material [47], and performed the statistical analysis on the photon emission number distribution. S. M. independently considered the influence of the radiative decay on the single-photon emission spectrum for $\chi<10$, joined the project in 2016, and pointed out the peak suppression in the multiphoton regime. The manuscript was written equally by both authors, with simulations and figures by M. T.
[1] V. Shiltsev and F. Zimmermann, Modern and future colliders, Rev. Mod. Phys. 93, 015006 (2021).

[2] M. Tanabashi et al. (Particle Data Group Collaboration), Review of particle physics, Phys. Rev. D 98, 030001 (2018).

[3] B. Badelek et al., The photon collider at TESLA, Int. J. Mod. Phys. A 19, 5097 (2004).

[4] P. Lebrun, L. Linssen, A. Lucaci-Timoce, D. Schulte, F. Simon, S. Stapnes, N. Toge, H. Weerts, and J. Wells, The CLIC programme: Towards a staged $e^{+} e^{-}$linear collider exploring the terascale: CLIC conceptual design report, CERN Yellow Reports: Monographs (CERN, Geneva, 2012) comments: 84 pages, published as CERN Yellow Report, https://cdsweb.cern.ch/record/1475225.

[5] R. J. Noble, Beamstrahlung from colliding electron-positron beams with negligible disruption, Nucl. Instrum. Methods Phys. Res., Sect. A 256, 427 (1987).

[6] V.I. Telnov, Problems in obtaining $\gamma \gamma$ and $\gamma e$ colliding beams at linear colliders, Nucl. Instrum. Methods Phys. Res., Sect. A 294, 72-92 (1990).

[7] P. Chen, Differential luminosity under multiphoton beamstrahlung, Phys. Rev. D 46, 1186 (1992).

[8] K. Yokoya and P. Chen, Beam-beam phenomena in linear colliders, in Frontiers of Particle Beams: Intensity Limitations, Lect. Notes Phys. Vol. 400 (Springer, New York, 1992), pp. 415-455, https://doi.org/10.1007/3-540-55250$2 \_37$.

[9] J. Esberg, U. I. Uggerhøj, B. Dalena, and D. Schulte, Strong field processes in beam-beam interactions at the Compact Linear Collider, Phys. Rev. Accel. Beams 17, 051003 (2014).

[10] V. I. Ritus, Quantum effects of the interaction of elementary particles with an intense electromagnetic field, J. Russ. Laser Res. 6, 497 (1985).
[11] V. N. Baier, V. M. Katkov, and V. M. Strakhovenko, Electromagnetic Processes at High Energies in Oriented Single Crystals (World Scientific, Singapore, 1998).

[12] A. Di Piazza, C. Müller, K. Z. Hatsagortsyan, and C. H. Keitel, Extremely high-intensity laser interactions with fundamental quantum systems, Rev. Mod. Phys. 84, 1177 (2012).

[13] W. Yan, C. Fruhling, G. Golovin, D. Haden, J. Luo, P. Zhang, B. Zhao, J. Zhang, C. Liu, M. Chen, S. Chen, S. Banerjee, and D. Umstadter, High-order multiphoton Thomson scattering, Nat. Photonics 11, 514 (2017).

[14] J. M. Cole et al., Experimental Evidence of Radiation Reaction in the Collision of a High-Intensity Laser Pulse with a Laser-Wakefield Accelerated Electron Beam, Phys. Rev. X 8, 011020 (2018).

[15] K. Poder et al., Experimental Signatures of the Quantum Nature of Radiation Reaction in the Field of an Ultraintense Laser, Phys. Rev. X 8, 031004 (2018).

[16] T. N. Wistisen, A. Di Piazza, H. V. Knudsen, and U. I. Uggerhøj, Experimental evidence of quantum radiation reaction in aligned crystals, Nat. Commun. 9, 795 (2018).

[17] V. I. Ritus, Radiative effects and their enhancement in an intense electromagnetic field, Sov. Phys. JETP 30, 1181 (1970), http://www.jetp.ras.ru/cgi-bin/dn/e_030_06_1181.pdf.

[18] N. B. Narozhny, Expansion parameter of perturbation theory in intense-field quantum electrodynamics, Phys. Rev. D 21, 1176 (1980).

[19] A. Fedotov, Conjecture of perturbative QED breakdown at $\alpha \chi^{2 / 3} \gtrsim 1$, J. Phys. 826, 012027 (2017).

[20] T. Podszus and A. Di Piazza, High-energy behavior of strong-field QED in an intense plane wave, Phys. Rev. D 99, 076004 (2019). 
[21] A. Ilderton, Note on the conjectured breakdown of QED perturbation theory in strong fields, Phys. Rev. D 99, 085002 (2019).

[22] A. Di Piazza and M. A. Lopez-Lopez, One-loop vertex correction in a plane wave, Phys. Rev. D 102, 076018 (2020).

[23] A. A. Mironov, S. Meuren, and A. M. Fedotov, Resummation of QED radiative corrections in a strong constant crossed field, Phys. Rev. D 102, 053005 (2020).

[24] V. Yakimenko, S. Meuren, F. Del Gaudio, C. Baumann, A. Fedotov, F. Fiuza, T. Grismayer, M. J. Hogan, A. Pukhov, L. O. Silva, and G. White, Prospect of Studying Nonperturbative QED with Beam-Beam Collisions, Phys. Rev. Lett. 122, 190404 (2019).

[25] T. G. Blackburn, A. Ilderton, M. Marklund, and C. P. Ridgers, Reaching supercritical field strengths with intense lasers, New J. Phys. 21, 053040 (2019).

[26] C. Baumann, E. N. Nerush, A. Pukhov, and I. Yu. Kostyukov, Probing non-perturbative QED with electron-laser collisions, Sci. Rep. 9, 9407 (2019).

[27] C. Baumann and A. Pukhov, Laser-solid interaction and its potential for probing radiative corrections in strong-field quantum electrodynamics, Plasma Phys. Controlled Fusion 61, 074010 (2019).

[28] A. Di Piazza, T. N. Wistisen, M. Tamburini, and U. I. Uggerhøj, Testing Strong Field QED Close to the Fully Nonperturbative Regime Using Aligned Crystals, Phys. Rev. Lett. 124, 044801 (2020).

[29] M. Aicheler et al., The compact linear collider (CLIC) Project implementation plan, arXiv:1903.08655.

[30] P. Bambade et al., The international linear collider: A global project, arXiv:1903.01629.

[31] I. F. Ginzburg, G. L. Kotkin, V. G. Serbo, and V. I. Telnov, Production of high-energy colliding $\gamma \gamma$ and $\gamma e$ beams with a high luminosity at VLEPP accelerators, JETP Lett. 34, 491 (1981), http://jetpletters.ru/ps/1533/article_23442.pdf.

[32] J. Gronberg, The photon collider, Rev. Accel. Sci. Techol. 07, 161 (2014).

[33] T. Takahashi, Future prospects of Gamma-Gamma collider, Rev. Accel. Sci. Techol. 10, 215 (2019).

[34] V. Telnov, Principles of photon colliders, Nucl. Instrum. Methods Phys. Res., Sect. A 355, 3 (1995).

[35] R. Blankenbecler and S. D. Drell, Quantum Beamstrahlung: Prospects for a Photon-Photon Collider, Phys. Rev. Lett. 61, 2324 (1988).

[36] F. Del Gaudio, T. Grismayer, R. A. Fonseca, W. B. Mori, and L. O. Silva, Bright $\gamma$ rays source and nonlinear BreitWheeler pairs in the collision of high density particle beams, Phys. Rev. Accel. Beams 22, 023402 (2019).

[37] V. N. Baier, V. M. Katkov, and V. M. Strakhovenko, Quantum radiation theory in inhomogeneous external fields, Nucl. Phys. B328, 387 (1989).

[38] A. Di Piazza, M. Tamburini, S. Meuren, and C. H. Keitel, Implementing nonlinear Compton scattering beyond the local-constant-field approximation, Phys. Rev. A 98, 012134 (2018).

[39] A. Ilderton, B. King, and D. Seipt, Extended locally constant field approximation for nonlinear Compton scattering, Phys. Rev. A 99, 042121 (2019).
[40] A. Di Piazza, M. Tamburini, S. Meuren, and C. H. Keitel, Improved local-constant-field approximation for strongfield QED codes, Phys. Rev. A 99, 022125 (2019).

[41] NIST Handbook of Mathematical Functions, edited by F. W. J. Olver, D. W. Lozier, R. F. Boisvert, and C. W. Clark (Cambridge University Press, Cambridge, England, 2010).

[42] J. Esberg and U. I. Uggerhøj, Does experiment show that beamstrahlung theory - strong field QED - can be trusted?, J. Phys. 198, 012007 (2009).

[43] S. S. Bulanov, C. B. Schroeder, E. Esarey, and W. P. Leemans, Electromagnetic cascade in high-energy electron, positron, and photon interactions with intense laser pulses, Phys. Rev. A 87, 062110 (2013).

[44] C. Rimbault, P. Bambade, O. Dadoun, G. Le Meur, F. Touze, M. del C. Alabau, and D. Schulte, GUINEA-PIG++: an upgraded version of the linear collider beam-beam interaction simulation code GUINEA-PIG, in Proceedings of the 2007 IEEE Particle Accelerator Conference (PAC) (IEEE, New York, 2007), pp. 2728-2730, http://hal.in2p3 .fr/in2p3-00200688/document.

[45] A. Gonoskov, S. Bastrakov, E. Efimenko, A. Ilderton, M. Marklund, I. Meyerov, A. Muraviev, A. Sergeev, I. Surmin, and E. Wallin, Extended particle-in-cell schemes for physics in ultrastrong laser fields: Review and developments, Phys. Rev. E 92, 023305 (2015).

[46] M. Tamburini, A. Di Piazza, and C. H. Keitel, Laser-pulseshape control of seeded QED cascades, Sci. Rep. 7, 5694 (2017).

[47] See Supplemental Material at http://link.aps.org/ supplemental/10.1103/PhysRevD.104.L091903 for (i) exact three dimensional (3D) analytical solution of Maxwell's equations for a finite size charge beam; (ii) details of the employed Monte Carlo simulation technique; (iii) details of the derivation of the multiphoton emission distribution.

[48] V. Yakimenko, L. Alsberg, E. Bong, G. Bouchard, C. Clarke, C. Emma, S. Green, C. Hast, M. J. Hogan, J. Seabury, N. Lipkowitz, B. OShea, D. Storey, G. White, and G. Yocky, FACET-II facility for advanced accelerator experimental tests, Phys. Rev. Accel. Beams 22, 101301 (2019).

[49] S. Redaelli, Stabilization of nanometre-size particle beams in the final focus system of the Compact Linear Collider (CLIC), Report No. CERN-AB-2004-026-ABP, 2003, https://cds.cern.ch/record/740518.

[50] J. Yan, Y. Yamaguchi, Y. Kamiya, S. Komamiya, M. Oroku, T. Okugi, N. Terunuma, K. Kubo, T. Tauchi, and J. Urakawa, Measurement of nanometer electron beam sizes with laser interference using Shintake monitor, Nucl. Instrum. Methods Phys. Res., Sect. A 740, 131 (2014).

[51] E. Marin, A. Latina, R. Toms, and D. Schulte, Final focus system tuning studies towards compact linear collider feasibility, Phys. Rev. Accel. Beams 21, 011003 (2018).

[52] T. G. Blackburn, D. Seipt, S. S. Bulanov, and M. Marklund, Radiation beaming in the quantum regime, Phys. Rev. A 101, 012505 (2020).

[53] S. Meuren and A. Di Piazza, Quantum Electron SelfInteraction in a Strong Laser Field, Phys. Rev. Lett. 107, 260401 (2011).

[54] S. Meuren, Nonlinear quantum electrodynamic and electroweak processes in strong laser fields, Ph.D. thesis, 
Ruprecht-Karls-Universität, Heidelberg, 2015, https://doi .org/10.11588/heidok.00018971.

[55] V. Dinu, T. Heinzl, and A. Ilderton, Infrared divergences in plane wave backgrounds, Phys. Rev. D 86, 085037 (2012).

[56] T. Podszus and A. Di Piazza, First-order strong-field QED processes including the damping of particles states, Phys. Rev. D 104, 016014 (2021).

[57] C. Itzykson and J. B. Zuber, Quantum Field Theory (Dover, New York, 2005).

[58] R. J. Glauber, Some notes on multiple-boson processes, Phys. Rev. 84, 395 (1951).

[59] A. Di Piazza, K. Z. Hatsagortsyan, and C.H. Keitel, Quantum Radiation Reaction Effects in Multiphoton Compton Scattering, Phys. Rev. Lett. 105, 220403 (2010).
[60] T. Barklow, Z. Huang, A. Naji, E. Nanni, J. Rosenzweig, A. Sakdinawat, S. Tantawi, and G. White, An X-ray FELbased $\gamma \gamma$ Collider Higgs Factory-Material for AF03 Summary Report (2020), https://indico.fnal.gov/event/ 46832/contributions/203937/attachments/138326/173436/ AF03_Report_XFEL_based_Gamma_Gamma_Collider .pdf.

[61] I. F. Ginzburg and G. L. Kotkin, Photon collider for energies 1-2 TeV, Phys. Part. Nuclei 52, 899912 (2021).

[62] D. Seipt and B. King, Spin- and polarization-dependent locally-constant-field-approximation rates for nonlinear Compton and Breit-Wheeler processes, Phys. Rev. A 102, 052805 (2020). 